\title{
Histological findings in the Paterson-Kelly syndrome
}

\author{
C. C. ENTWISTLE AND A. JACOBS \\ From the Institute of Pathology, Welsh National School of Medicine, Cardiff
}

SYNOPSIS Forty-one specimens taken from lesions in the post-cricoid region of patients with the Paterson-Kelly syndrome have been examined. Most webs consist of normal oesophageal mucosa $\vec{\omega}$ which may be associated with underlying muscle and nerve lesions. Strictures also show a chronic $\frac{?}{8}$ non-specific inflammation often with epithelial changes and sometimes squamous carcinoma. Other lesions observed clinically have also shown malignant change.

This paper describes the histological features of specimens taken from apparently benign lesions in the post-cricoid region of patients with the PatersonKelly syndrome. The lesions were clearly defined by oesophagoscopy and usually also radiologically. We have deliberately excluded cases of clinically obvious hypopharyngeal carcinoma.

\section{MATERIAL AND METHODS}

Forty-one specimens were obtained from post-cricoid lesions in 39 patients. The specimens were mostly small and contained epithelium and subepithelial elements superficial to the main muscular layers. In five instances, the biopsies consisted of epithelium alone. Deeper tissues were present in specimens from four necropsies, but in only one biopsy.

Paraffin sections were stained by haematoxylin and eosin, periodic acid-Schiff (P.A.S.) for the detection of glycogen with control sections pretreated with salivary diastase at $37^{\circ} \mathrm{C}$. for non-glycogen polysaccharides; Perls's reaction to identify haemosiderin, and van Gieson's stain for collagen. In post-mortem tissue, Luxolvan Gieson's stain was also used to demonstrate nerve and muscle changes.

Arrangement of the material into the following three groups facilitates interpretation of the histological appearances in the different lesions.

WEBS Twenty-six specimens were derived from postcricoid webs. The 22 women and two men were aged 29 to 74 years (mean 53.9 years) and their mean duration of dysphagia was 9.0 years. Nineteen patients had never required dilatation; five had had one previous dilatation, and the group includes as separate specimens tissues taken on the first occasion in two of these. All but four patients were anaemic either at the time of biopsy or before then.

STRICTURES There were nine women with upper oesoReceived for publication 1 December 1964. phageal strictures. The age range was 51 to 80 years (mean 63.3 years). The mean duration of dysphagia was $18 \cdot 1$ years. Three patients had had one, five, and seven dilatations respectively. Anaemia was present in seven patients.

MISCELLANEOUS LESIONS Although oesophagoscopy revealed three webs and one mild stricture, this group con-O cerns other post-cricoid abnormalities found in six women aged 58 to 74 years (mean 66.4 years). While one of these had dysphagia for only six months, the others had had the symptom for over 20 years. The patient with the short history had a small ulcer $18 \mathrm{~cm}$. from the in- $\vec{F}$ cisors. A biopsy was taken from this and from a "white $\frac{\text { 을 }}{3}$ plaque of thickened mucosa' seen in three other women. In two, these were just proximal to coexistent webs, the third had no other lesion.

In one patient an apparently normal piece of mucosan just above a web (included in the first group) was biopsied:and in another a fold of mucosa in the post-cricoid area was taken, this being the only unusual feature seen.

\section{HISTOLOGY}

WEBS Figure 1 shows a section through a typical post-cricoid web. The appearance is essentially thate of a fold of normal oesophageal epithelium with some underlying loose connective tissue. In three of the 26 specimens there was a minor degree of irregularity in the basal cells, including the presence of hyperchromatic nuclei and an increase in mitotice activity. Two of these showed in addition a loss of glycogen from the prickle cells, and, in one case, an abnormal diastase-resistant polysaccharide was present in the superficial squamous cells. Absence of glycogen was noted in three other cases.

The subepithelial tissue was insufficient for? comment in four specimens, and in six was entirely normal. In eight specimens a few chronic inflam? matory cells and polymorphs were present, and in a 


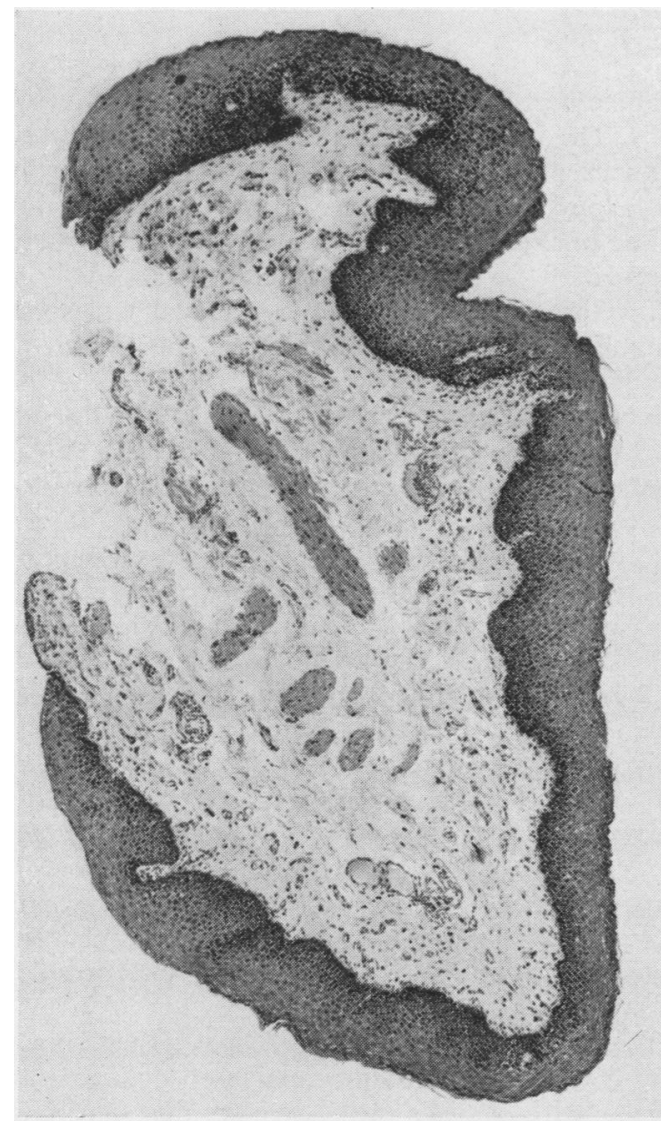

FIG. 1.

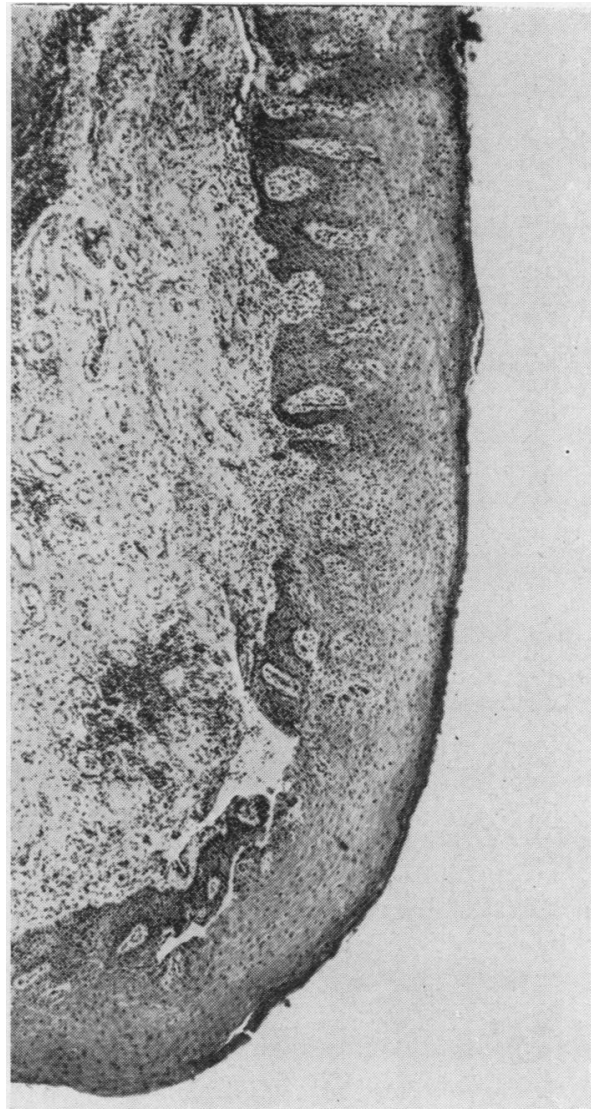

FIG. 2.

FIG. 1. Post-cricoid web. Haematoxylin and eosin $\times 50$.

FIG. 2. Post-cricoid web showing chronic inflammation. Haematoxylin and eosin $\times 70$.

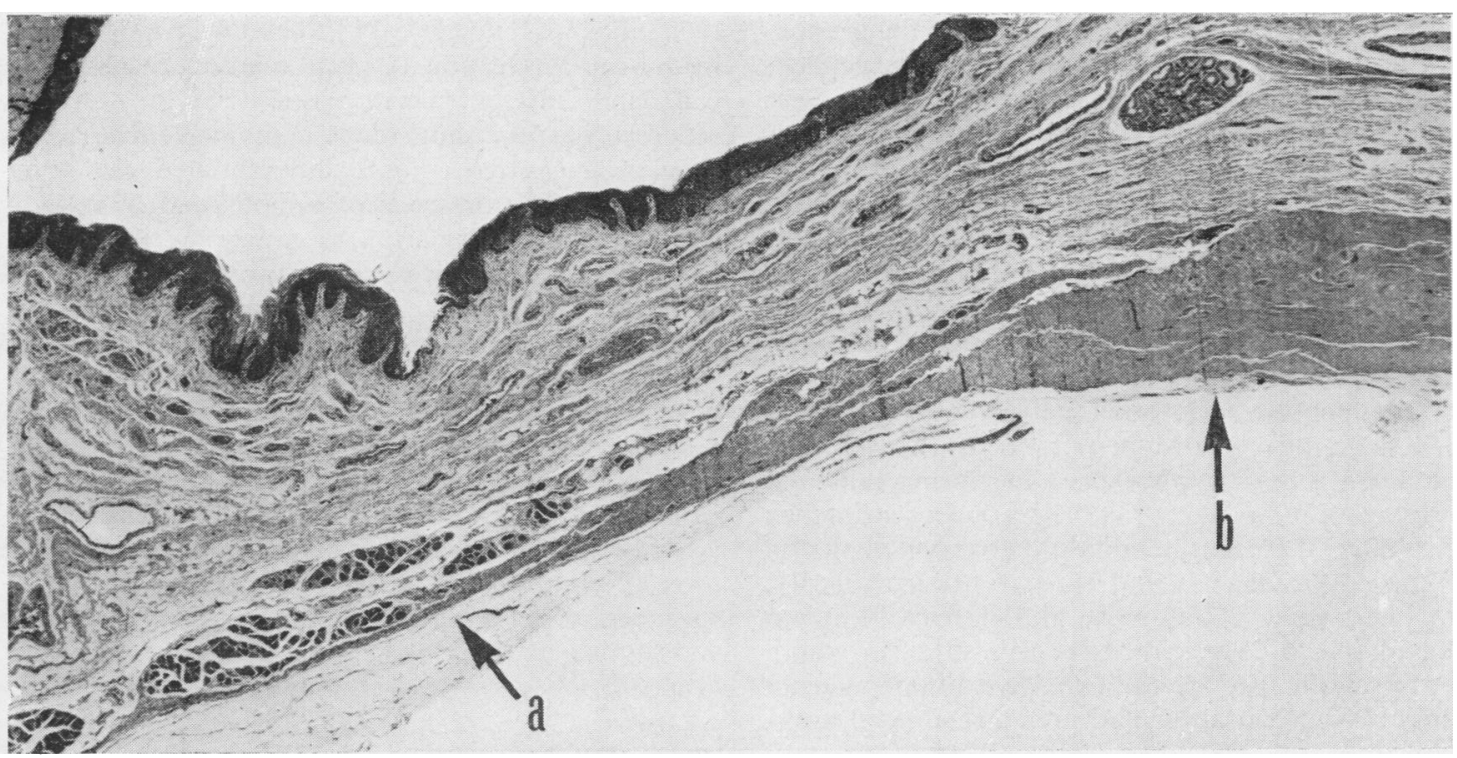

FIG. 3. Transverse section of anterior wall of oesophagus adjacent to web showing surviving muscle bundles (a) laterally and necrotic muscle with fibrosis (b) centrally. Haematoxylin and eosin $\times 30$. 


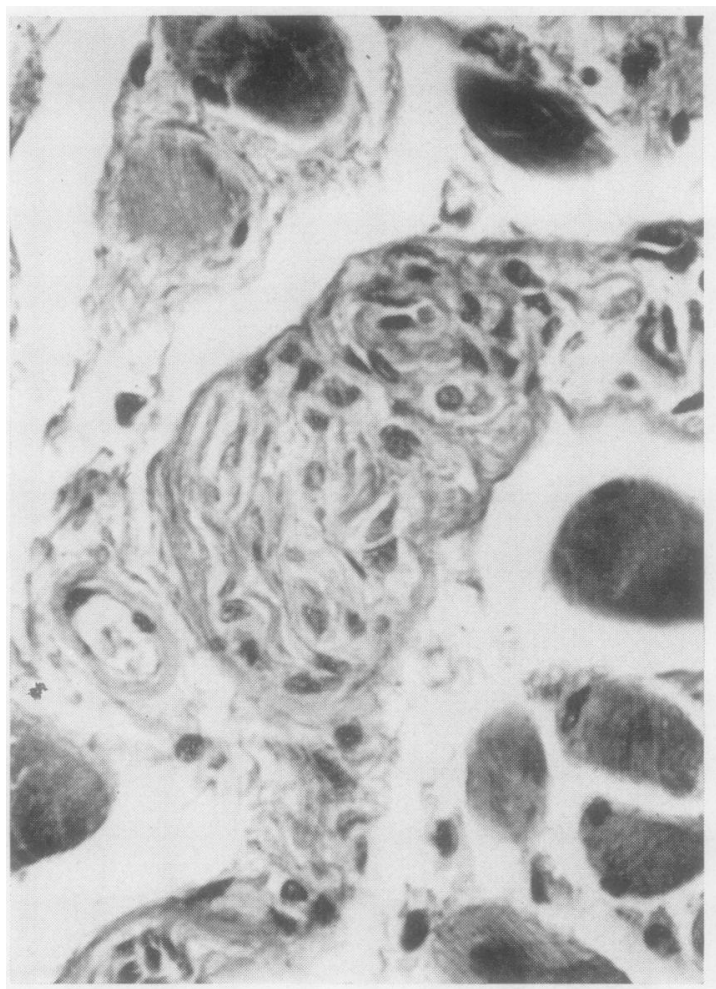

FIG. 4. Nerve showing loss of myelin. Luxol and van Gieson $\times 520$.

further seven there were considerable numbers of lymphocytes and plasma cells (Fig. 2); in five of the latter cases there was also some inflammatory infiltration of the epithelium. Seven specimens showed increased collagen coexistent in six with cellular infiltration. Haemosiderin deposits were found in three webs.

Two of the patients had biopsies taken at two different times. In one, whose dysphagia had been present for over 15 years, the first specimen showed only a very few inflammatory cells under the epithelium. The second specimen obtained a year later showed gross deposits of haemosiderin but no sign of inflammation. The other patient had symptoms for less than five years when first seen; the web then contained a few lymphocytes and plasma cells and an increase in collagen. On the second occasion, six years later, there was in addition, glycogen depletion of the epithelial cells and some basal irregularity. No haemosiderin was seen, and it may be noted that of the three specimens in this study in whom haemosiderin was found, two were obtained from patients who had never had previous surgical interference.
Three necropsies were performed on patients with webs. The lamina propria contained a moderate or. slight excess of lymphocytes and plasma cells. The epithelium of the web in two of them was normal buf? in one of these cases there was atrophy and nucleat irregularity with glycogen depletion about $1 \mathrm{~cm}=$ below the web. The third case showed widespreacp epithelial atrophy with depletion of prickle-celP glycogen.

ब)

In all three specimens the main longitudinal striped 5 muscle, which is normally thin anteriorly, showeddegenerative changes most marked in the region of $\vec{\omega}$ the web. In one, a large portion of this anterior muscle (Fig. 3) was necrotic but without surrounding inflammation $(b)$. The rest of the striped muscle inoo this case showed varying degrees of atrophy, often of individual fibres within bundles, together with re $-\hat{D}^{-}$ duplication of sarcolemmal nuclei and replacement $5^{\infty}$ collagenosis but without myositis as such $(a)$. In? many small nerve branches, there was loss of myelin with increase of collagen (Fig. 4). There was nof endarteritis or occlusion of the small vessels seen

STRICTURES All the specimens from strictures in the pharyngo-oesophageal region revealed some histological abnormality, as might be expected from thes gross appearances.

The only biopsy containing deeper tissues showect mild chronic inflammation, increased collagen, and $\mathbb{B}$ degenerative changes in the striped muscle (Fig. 5) $\vec{\Rightarrow}$ These consisted of marked variation in size and stainability of fibres, some being atrophic, others? greatly swollen, some having central nuclei, anđō some with nuclear proliferation. The small nerves seen appeared normal, but there was a focus of round-cell infiltration in close relation to ganglion cells and adjacent nerve tissue (Fig. 6). Deeper sections from the same block contained foci of cellso strongly suggestive of poorly differentiated squamous carcinoma. The presence of tumour and of round-을 cell infiltration in relation to ganglionic tissue was confirmed in a further biopsy four months later.

In two specimens epithelial abnormalities were found consisting of acanthosis and basal irregularity hyperchromatic nuclei, increased mitotic activity and glycogen depletion. In all strictures the subepitheliak tissue contained lymphocytes and plasma cells and? a few polymorphs. In most cases, this inflammatorye reaction also involved the epithelium, though to as lesser extent. Increased collagen was present in ${ }^{+}$ three specimens. No haemosiderin was found in any $\frac{0}{3}$ specimen. The presence of inflammatory reaction $\frac{\mathrm{D}}{\mathrm{D}}$ and fibrosis does not seem to be related to the dura- $\frac{\stackrel{\rho}{D}}{\bar{D}}$ tion of dysphagia or to preceding dilatation.

MiSCELLANEOUS LESIONS Two of the clinically ob- $\Omega$ 


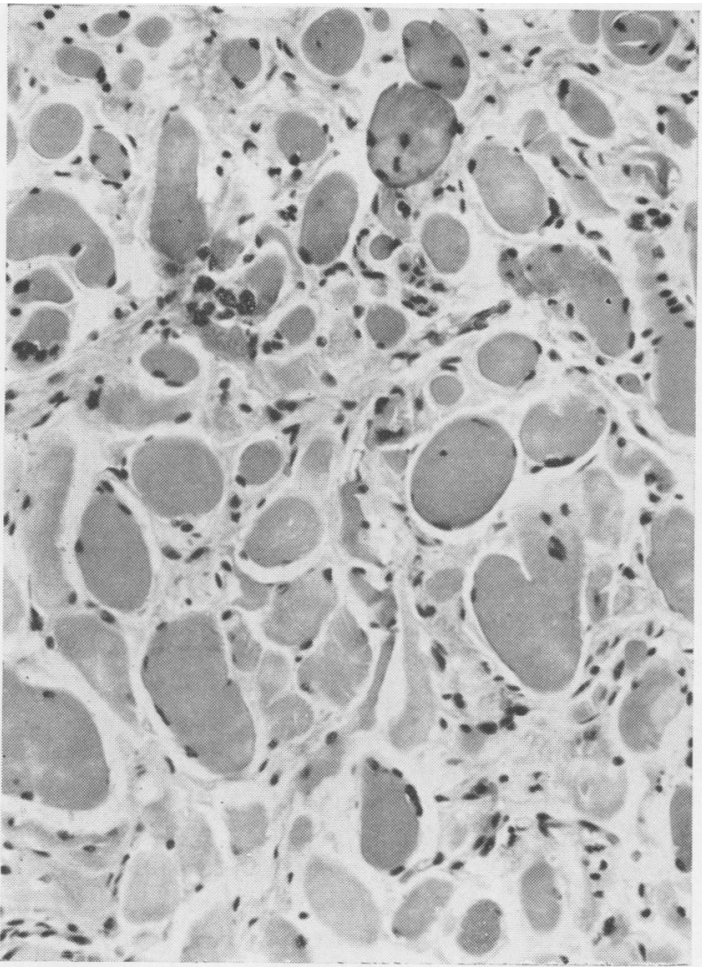

FIG. 5 .

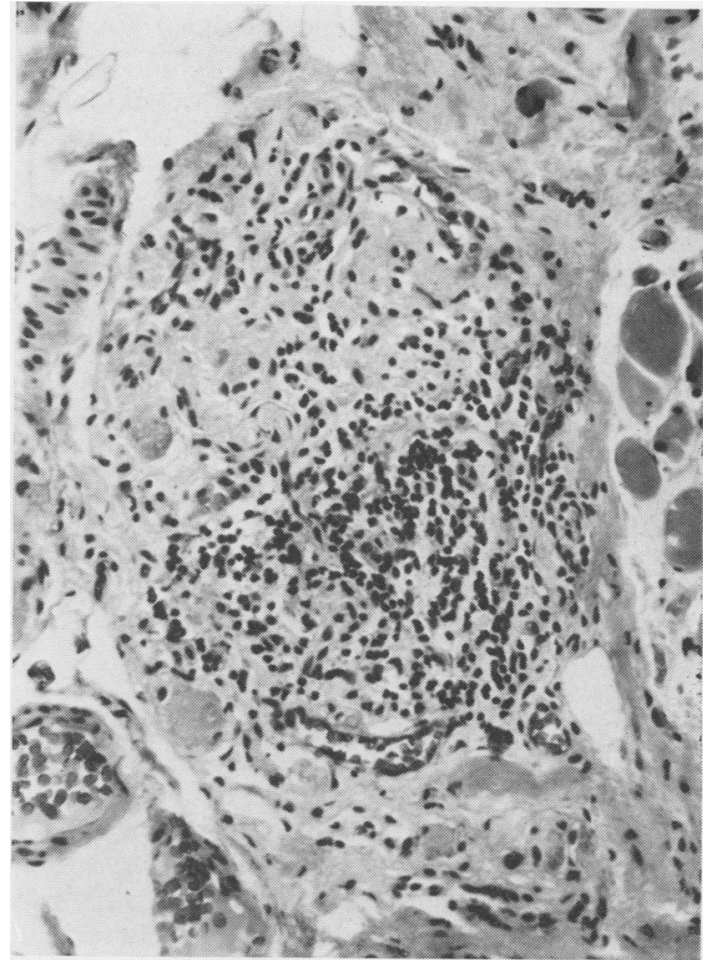

FIG. 6.

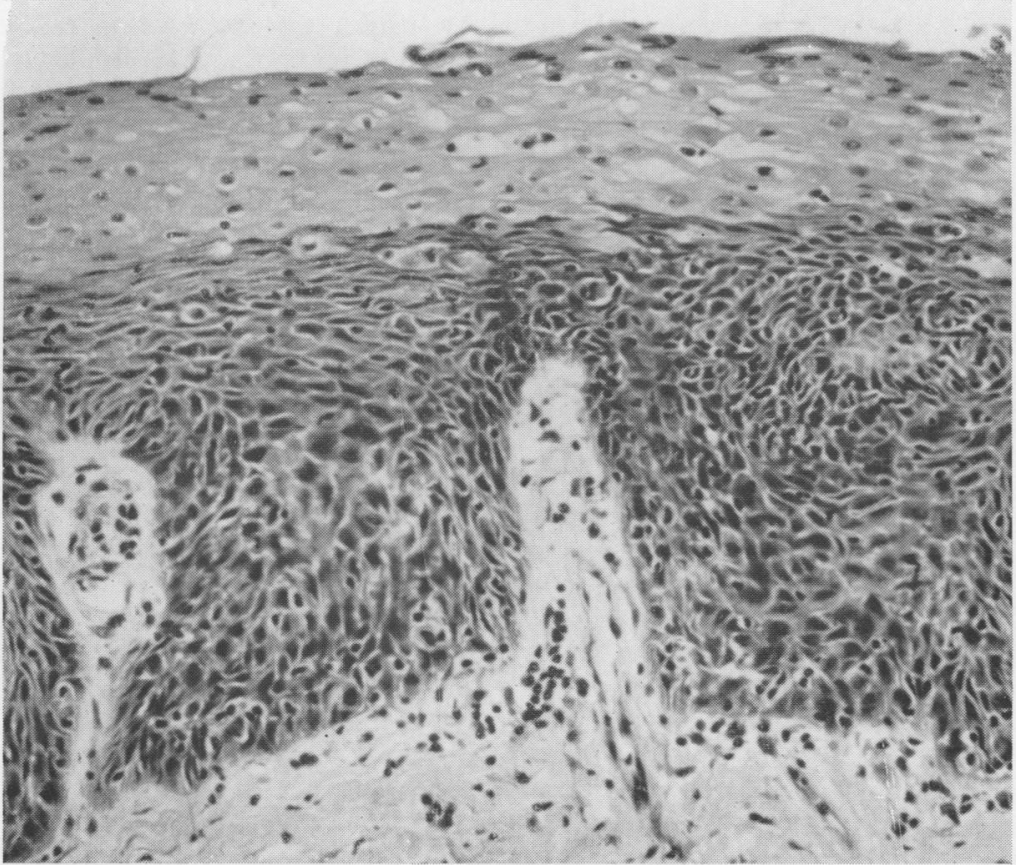

FIG. 5. Striped muscle in a stricture showing abnormalities in some fibres (see text). Haematoxylin and eosin $\times 186$.

FIG. 6. Round cell infiltration around nervous tissue. Haematoxylin and eosin $\times 186$.

FIG 7. Mucosa showing carcinoma in situ. Haematoxylin and eosin $\times 186$.

FIG. 7 . 
served thickened white plaques of mucosa showed unmistakable carcinoma in situ (Fig. 7), but the third consisted of apparently normal tissue and the patient is still well four years later with no further surgical treatment.

Histological examination of the small postcricoid ulcer present after only six months of dysphagia revealed non-specific changes including some irregularity and hyperchromatism of the epithelial nuclei with increased mitotic activity and inflammation, including polymorphs as well as lymphocytes and plasma cells. The specimen taken from a prominent mucosal fold in another patient showed similar epithelial changes but with less marked inflammation. The mucosal specimen taken from above a web had a mild chronic inflammatory infiltrate while the web itself consisted of entirely normal oesophageal mucosa.

\section{DISCUSSION}

The commonest type of lesion in patients with the Paterson-Kelly syndrome is a post-cricoid web. Benign upper oesophageal strictures are also seen. There is an impression that strictures occur in patients with long-standing disease and that malignant change in the epithelium may eventually be found in 10 to $30 \%$ of cases (Jones, 1961). Some authors have described thinning of the epithelium (Goldstein, 1963), while others have observed thickening and hyperkeratinization (Suzman, 1933).

Suzman (1933) performed a necropsy on a woman of 48 with a stricture who died from mediastinitis following perforation of the pharynx by a bougie. He described the epithelium as having an appearance somewhat similar to leukoplakia and the submucosa as showing a moderate degree of infiltration with mononuclear cells, chiefly lymphocytes and plasma cells. The deep muscle was said to have 'hyaline areas suggestive of Zenker's degeneration'. There was no abnormality in Auerbach's plexus. Hoover (1935) described a biopsy from a web as 'two layers of mucous membrane with a small amount of fibrous tissue between them'. Savilahti (1946) reported on a woman of 71 who had prolonged difficulty in swallowing due to 'food sticking in the upper part of the oesophagus'. At necropsy there was no apparent obstruction in the pharyngooesophageal region. The mucosa here was 'smooth and thinner than usual' and was histologically atrophic with some nuclear irregularities. The muscle layer was normal at this point, but in the mid-oesophagus where the epithelial changes were similar to those higher up, there was a $5 \mathrm{~cm}$. segment where the muscle was atrophic. Shamma'a and Benedict (1958) report that in five biopsies, four from the upper and one from the mid-oesophagus, $\frac{0}{\vec{\sigma}}$ there was normal squamous epithelium, but the underlying connective tissue was 'the seat of a mild $\Rightarrow$ : chronic inflammation with mononuclear cells. Lym- $\frac{\mathcal{O}}{+}$ phoid hyperplasia with lymphoid nodular formation was noted in one case'.

One of the difficulties in evaluating the PatersonKelly syndrome is in assessing which features are primarily due to iron-deficiency and which are inde- \& pendent of it. Most patients by the time they have $\vec{\circ}$ developed a lesion in the pharynx are suffering from iron deficiency which by itself can lead to epithelial $\vec{\omega}$ changes in the mouth (Jacobs, 1960; McLean Baird, Dodge, Palmer, and Wawman, 1961) and probably in the post-cricoid region (Jacobs, 1961). $\vec{\infty}$ These are essentially atrophy, glycogen depletion, $\rightarrow$ and occasional abnormal keratinization. Changes in $\vec{c}$ the subepithelial tissue are common and consist $\infty$ mainly of a minor round-cell infiltration. In the 9 present series obvious atrophy was present in only two women. Both had recently been anaemic but $\subseteq$ abnormal keratinization was not seen.

The outstanding histological features of the lesions $\vec{\bullet}$ in the Paterson-Kelly syndrome are first the essen- $f$ tially 'normal' epithelium in most post-cricoid webs, secondly the presence of a basal-cell irregularity, in situ and frankly invasive carcinoma in many of the other lesions; thirdly, the marked inflammatory reaction in the lamina propria, and fourthly, the $\stackrel{\mathbb{D}}{\circ}$ presence of degenerative changes in striped muscle and nerves in the few instances where assessment has been possible. It has not been possible to implicate any specific factor in the pathogenesis of the lesions. The difficulty of distinguishing between primary and secondary iron deficiency in relation to the dysphagia ? means that the aetiological importance of this factor cannot be assessed.

It is possible that repeated dilatation could be important in producing local trauma followed by inflammation and fibrosis. The main reason for stain- 음 ing all specimens by Perls's reaction was to detect $\rightarrow$ the remains of old haemorrhage induced in this way, but haemosiderin was detected in only three webs N and in no other lesions. Of the three, one had been subjected to dilatation a year earlier and the biopsy on that occasion contained no stainable iron. The $\omega$ two other patients had never required surgical interference. The absence of haemosiderin in a web 6 known to have been the site of biopsy six years $\Phi$ earlier indicated that either the signs of haemorrhage $\stackrel{?}{\rightarrow}$ may disappear altogether, or simply that biopsy is 0 subject to sampling error. The presence of excess collagen in the webs was not related to previous dilatation, nor in general was an inflammatory $\stackrel{\unrhd}{\unrhd}$ reaction, though two webs in which lymphoid follicles were found had both been dilated previously. 
Trauma from the passage of food is an unknown factor. Only three patients of the nine with strictures had had oesophageal instrumentation, indicating that this is not an essential factor in the evolution of the lesion. Two of these patients had 12 dilatations between them.

Only four necropsy specimens and a single biopsy allowed examination of the main oesophageal muscle, Auerbach's plexus, and small nerve branches. There was no evidence of Zenker's degeneration as described by Suzman (1933); the severe infection and terminal cachectic state in his patient may account for this phenomenon. No sign of vascular insufficiency was found to explain the presence of infarcted muscle in the one specimen where this was seen. All these five specimens showed some degree of peripheral neuropathy, the aetiology of which was not obvious. The round-cell infiltration seen in one biopsy and its repeat four months later may perhaps be part of a carcinomatous neuropathy which may occur up to three years before a tumour becomes overt (Brain and Henson, 1958).

In the current series no web showed carcinoma though this was common in other post-cricoid lesions. No evidence has accrued that a transition from web to stricture occurs, and it may be that these are different types of lesion both in origin and evolution, for patients are often seen where the two lesions coexist at different levels in the pharyngooesophageal region. The web appears to be an accentuation of a normal mucosal fold with occasional minor secondary epithelial or subepithelial changes. It does not seem to be caused by muscle spasm, and only in a few instances is there fibrosis which might be expected to perpetuate the lesion. Studies of the underlying muscle are few and confined largely to necropsy material, but the changes described here could initiate a localized dysfunction of this part of the oesophagus.

The strictures are invariably associated with inflammation and the absence of haemosiderin from all nine specimens makes this unlikely to be traumatic in origin.

The clinical implications of the present study are that the biopsy of webs is unlikely to reveal the onset of a carcinoma in patients with the Paterson-Kelly syndrome, but that histological examination of other lesions, especially after a long history of dysphagia, may disclose malignant disease.

We should like to thank Professor J. Gough for his criticism and advice, and the surgeons and pathologists through whose courtesy this material has been made available.

\section{REFERENCES}

Brain, R., and Henson, R. A. (1958). Lancet, 2, 971.

Goldstein, F. (1963). In Gastroenterology, vol. 1, 2nd ed., p. 216, edited by H. L. Bockus. Saunders, Philadelphia.

Hoover, W. B. (1935). New Engl. J. Med., 213, 394.

Jacobs, A. (1960). J. clin. Path., 13, 463.

- (1961). Brit. J. Cancer, 15, 736.

Jones, R. F. McN. (1961). J. Laryng., 75, 529.

McLean Baird, I., Dodge, O. G., Palmer, F. J., and Wawman, R. J. (1961). J. clin. Path., 14, 603.

Savilahti, M. (1946). Acta med. scand., 125, 40.

Shamma'a, M. H., and Benedict, E. B. (1958). New Engl. J. Med., 259, 378.

Suzman, M. M. (1933) Arch. intern. Med., 51, 1. 